\title{
ANÁLISE MÉTRICA DO FORAME DA MANDÍBULA EM CRÂNIOS SECOS
}

\section{Josiane Medeiros de Mello}

Centro de Ciências Biológicas, Departamento de Ciências Morfológicas, Universidade Estadual de Maringá (UEM), Maringá (PR), Brasil.

E-mail: jmedeirosmello@gmail.com

\section{Rodrigo Chicalski}

Odontólogo- Especialista em Anatomia e Histologia.

Célia Regina Godoy Gomes

Ana Paula Vidotti

Larissa Renata Oliveira Bianchi

Centro de Ciências Biológicas, Departamento de Ciências Morfológicas, Universidade Estadual de Maringá (UEM), Maringá (PR), Brasil.
RESUMO: No âmbito da odontologia, frequentemente são encontradas situações onde é essencial o uso da anestesia para que seja possível efetuar os procedimentos necessários. Em especial, a mandíbula requer um diferencial no quesito anestesia, pois sua cortical óssea não permite uma boa difusão dos sais anestésicos, fazendo com que seja necessário um bloqueio do nervo alveolar inferior. O objetivo dessa pesquisa foi mensurar a posição do forame da mandíbula em mandíbulas estabelecendo suas relações com pontos de referência do ângulo e do ramo da mandíbula, pois se entende que havendo variações anatômicas significativas pode-se justificar alguns erros no bloqueio anestésico do nervo alveolar inferior, seja direito ou esquerdo. Foram selecionadas mandíbulas de 48 indivíduos as quais a análise realizada constituiu-se da medida do forame da mandíbula em relação aos seguintes pontos: ponto (A) - condílico; ponto (B) - gônio; ponto (C) - borda anterior; e ponto (D) - borda posterior. As medidas do forame da mandíbula foram realizadas em ambos os antímeros, utilizando paquímetro digital (Western Pro), com resolução de $0,1 \mathrm{~mm}$. As medias encontradas para as distâncias analisadas foram: borda anterior direita $15,285 \mathrm{~mm}$ e para borda esquerda $15,537 \mathrm{~mm}$; para borda posterior direita $12,531 \mathrm{~mm}$ e para a borda posterior esquerda $12,593 \mathrm{~mm}$; para o côndilo direito $42,839 \mathrm{~mm}$ e para o côndilo esquerdo de $42,839 \mathrm{~mm}$; distância média para o ponto gônio direito foi de $20,762 \mathrm{~mm}$ e para o esquerdo de $20,725 \mathrm{~mm}$. Não foram encontradas variações significativas entre os antímeros analisados.

PALAVRAS-CHAVE: Anatomia; Forame mandibular; Mandíbula; Medida.

\section{METRIC ANALYSIS OF THE JAW FORAMEN IN DRY SKULLS}

ABSTRACT: There are several situations in Dentistry in which the use of anesthetics is relevant to undertake the required procedures. The jaw requires different treatments when the anesthetic issue is involved since the bony cortical does not allow a good diffusion of the anesthetic salts. Consequently, the blockage of the lower alveolar nerve is required. Current research measures the foramens in jaws and establishes their relationships with reference points of angle and jaw branch. If there are significant anatomical variations, errors in the anesthetic blockage of the lower alveolar right or left nerve may be justified. The jaws of 48 subjects were selected and jaw foramen was measured against the following sites: Site A - condylic; site B - gonic; site $\mathrm{C}$ - anterior edge; site $\mathrm{D}$ - posterior edge. Foramen measurements were undertaken in the two antimeres by digital caliper (Western Pro), $0.1 \mathrm{~mm}$ resolution. Mean distances analyzed were: right anterior edge $15.285 \mathrm{~mm}$ and left anterior edge $15.537 \mathrm{~mm}$; right posterior edge $12.531 \mathrm{~mm}$ and left posterior edge $12.593 \mathrm{~mm}$; right condyle 42.839 
$\mathrm{mm}$ and left condyle $42.839 \mathrm{~mm}$; mean distance to right gonic site was $20.762 \mathrm{~mm}$ and to left gonic site 20.725 $\mathrm{mm}$. No significant variations were reported between the antimeres.

KEYWORDS: Jaws; Jaw foramen; Measurement; Anatomy.

\section{INTRODUÇÃO}

A mandíbula é um osso ímpar, com uma cortical relativamente mais densa comparada com os ossos da maxila, que pode ser subdividia em: corpo e dois ramos, o direito e o esquerdo, delimitados pelo ângulo da mandíbula. Cada um dos ramos apresenta um processo coronóide, incisura mandibular, e um processo condilar, também conhecido como côndilo da mandíbula, que se encaixa na fossa mandibular do osso temporal, juntamente com a cápsula articular, formando a articulação temporomandibular (DI DIO, 2002; GARDNER; GRAY; O'RAHILLY, 1988; MADEIRA, 2010).

Em análise da parte lingual ou interna da mandíbula, pode-se verificar, dentre os muitos processos existentes, o forame da mandíbula que é de extrema importância para a anestesiologia, em especial na odontologia. Encontrado na parte interna cerca de 1 $\mathrm{cm}$ superior ao plano oclusal, logo após a língula da mandíbula no sentido anteroposterior, ele é o início do canal mandibular que atravessa toda a mandíbula do seu respectivo lado de entrada, até chegar à parte anterior da mandíbula, formando o forame mentual, sendo a porta de saída do nervo alveolar inferior. Este, por sua vez, tem sua entrada pelo forame da mandíbula, e é responsável pela inervação da mandíbula, dos dentes e da região do mento do seu respectivo lado (AMORIM, 2006; MADEIRA, 2010; SUAZO et al., 2007).

Para procedimentos invasivos nos dentes inferiores, faz-se necessário o bloqueio do nervo alveolar inferior, que tem o fundamento no perfeito conhecimento da região anatômica, sabendo o trajeto do tronco nervoso, onde será depositado o anestésico (VASCONCELOS et al., 2008). Entre os casos de insucessos do procedimento, cerca de 30 a 40\%, estão a falta de conhecimento das estruturas anatômicas e suas variações, além das razões patológicas, farmacológicas, fisiológicas e a técnica de administração (FERNANDEZ et al., 2005; QUEIROZ et al., 2015).

O objetivo desse estudo foi mensurar a posição do forame da mandíbula de ambos os lados, estabelecendo suas relações com pontos de referência do ângulo e do ramo da mandíbula, para saber se há variações anatômicas significativas que possam levar ao insucesso das técnicas de bloqueio do nervo alveolar inferior, pois se entende que havendo variações anatômicas significativas, estas podem justificar alguns erros no bloqueio anestésico do nervo alveolar inferior.

\section{METODOLOGIA}

Para o presente trabalho foram utilizadas 100 mandíbulas provenientes de esqueletos humanos exumados, previamente submetidos aos processos de limpeza em água corrente, clareamento com peróxido de hidrogênio e posterior impermeabilização com cola a base de PVA diluída em água (80\% cola/20\% água), do acervo do laboratório de Anatomia Humana do Departamento de Ciências Morfológicas da Universidade Estadual de Maringá, município de Maringá (Pr).

O método utilizado foi estudo descritivo e de observação, que analisou mandíbulas humanas secas. As amostras foram selecionadas considerando-se o bom estado de conservação das peças anatômicas, estado necessário para a mensuração do forame da mandíbula e confiabilidade dos dados. Foram selecionadas 48 mandíbulas, dentadas, semidentadas e adentadas, numeradas aleatoriamente para a sua identificação. Em cada mandíbula foi realizada a análise macroscópica, que consistiu da medida do forame da mandíbula em relação aos seguintes pontos: ponto (A) - condílico; ponto (B) gônio; ponto (C) - borda anterior; e ponto (D) - borda posterior, conforme esquema evidenciado por Minatel et al. (2008) na Figura 1. 


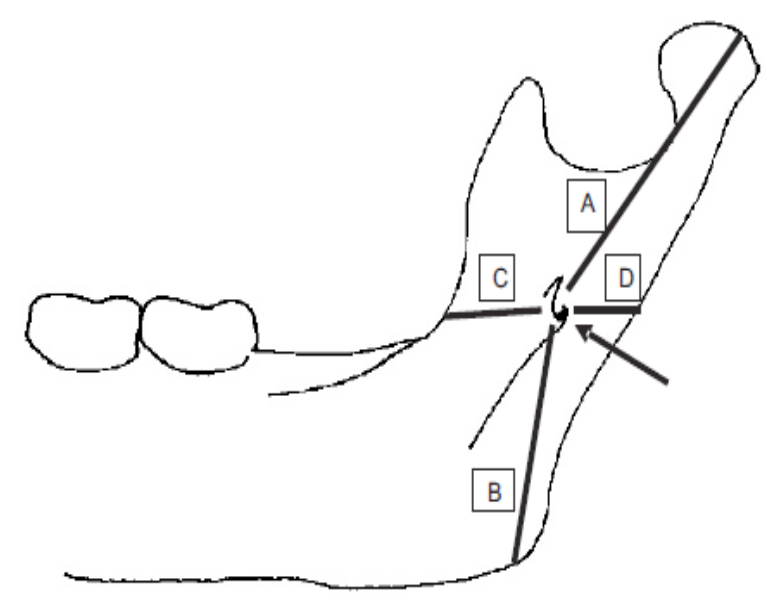

Figura1. Figura esquemática da mandíbula humana, sendo a distância do forame para: ponto (A) - condílico; ponto (B) - gônio; ponto (C) - borda anterior; e ponto (D) - borda posterior (MINATEL et al., 2008).

As medidas do forame da mandíbula foram realizadas em ambos os antímeros, utilizando paquímetro digital (Western Pro), com resolução de $0,1 \mathrm{~mm}$, tomando em conta os pontos da Figura 1, após indicado a maior medida, menor medida, média e mediana de todas as mandíbulas, foi realizado o teste estatístico ANOVA para significância estatística.

O presente estudo teve aprovação do Comitê Permanente de ética em pesquisa envolvendo seres humanos, da Universidade Estadual de Maringá, conforme parecer $n^{\circ} 223 / 2011$ de maio de 2011.

\section{RESULTADOS}

Os resultados obtidos mostraram que, no lado direito, obteve-se uma média de 15,285 mm em relação à borda anterior (ponto $\mathrm{C}$ ) e o forame da mandíbula direito, sendo a maior distância encontrada de 19,2 $\mathrm{mm}$ e a menor foi de $9,4 \mathrm{~mm}$. Para borda posterior (ponto $\mathrm{D}$ ), foi obtida uma média de $12,531 \mathrm{~mm}$, com a maior distância sendo $18,9 \mathrm{~mm}$ e a menor $9,2 \mathrm{~mm}$. Analisando a distância para o côndilo direito (ponto A), foi encontrada a média de $42,839 \mathrm{~mm}$, levando-se em consideração a maior distância como $51,4 \mathrm{~mm}$ e a menor de 32,1 mm. Para o gônio direito (ponto B), temse uma média de $20,762 \mathrm{~mm}$ evidenciando que a maior distância encontrada foi de 29,1 e a menor de 12,5 dentre as mandíbulas analisadas.

Os dados obtidos estão agrupados no gráfico mostrado na Figura 2.

Observa-se que, em todos os casos, os valores de média e mediana encontrados foram muito próximos entre si, o que significa que não houve uma distribuição assimétrica dos resultados. As medidas da borda posterior direita foram as que apresentaram menor valor de desvio padrão, o que significa que os resultados estão mais próximos da média. Por outro lado, a distância ao gônio foi a que apresentou o maior desvio padrão, mostrandose mais distante do valor médio obtido, que pode ser observado na Tabela 1.

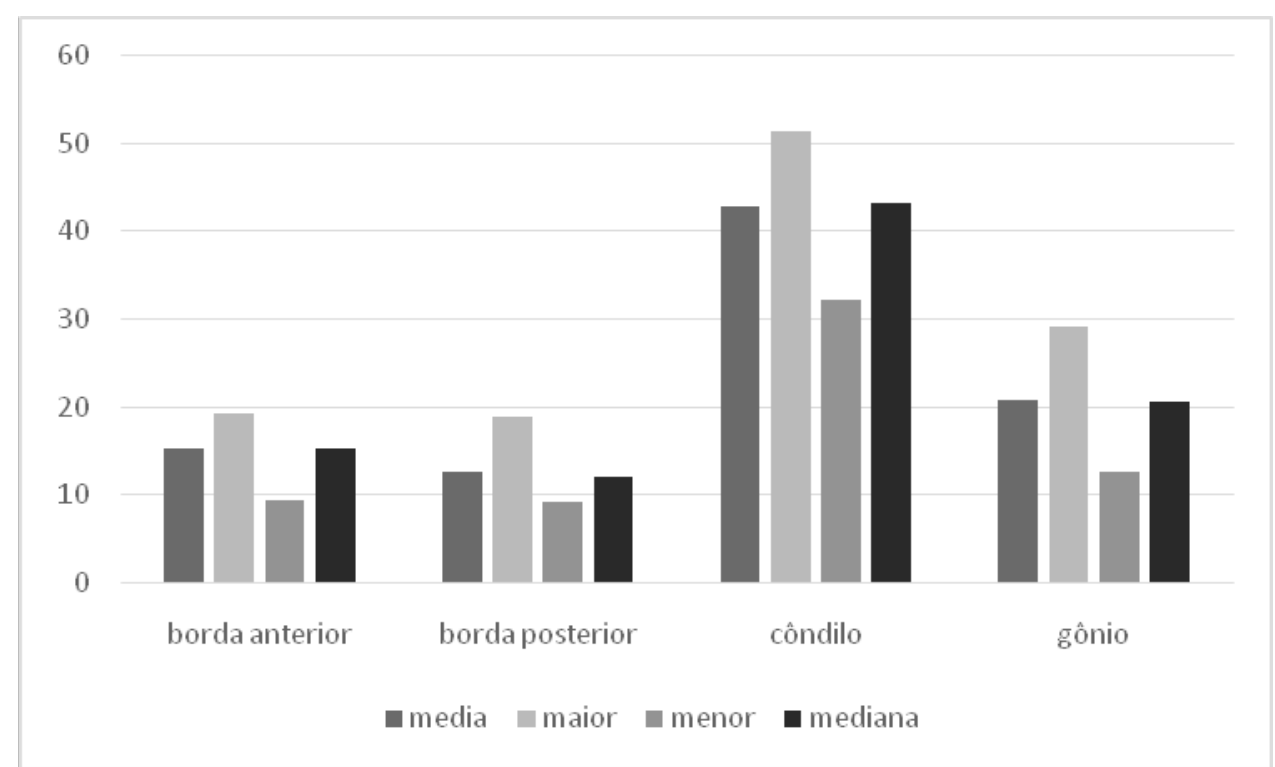

Figura 2. Resultados obtidos na medida do forame da mandíbula em relação ao côndilo, gônio, borda anterior e borda posterior do lado direito. 
Tendo em vista as medidas do lado esquerdo, obteve-se uma média do forame para borda anterior (ponto C) de $15,537 \mathrm{~mm}$, observando que a maior medida foi de $23,8 \mathrm{~mm}$ e a menor $9,4 \mathrm{~mm}$. A média encontrada entre a borda posterior (ponto D) foi de $12,593 \mathrm{~mm}$, sendo a maior medida entre as mandíbulas de $19,6 \mathrm{~mm}$ e a menor medida de $8,6 \mathrm{~mm}$. Observando-se a distância do forame da mandíbula esquerdo para o côndilo esquerdo (ponto A), observou-se uma média, entre as 48 mandíbulas medidas, de 42,662 mm, sendo que a maior distância foi de $49,8 \mathrm{~mm}$ e a menor de $31,7 \mathrm{~mm}$. Para a última medida, do forame da mandíbula esquerdo até $o$ ponto gônio esquerdo (ponto B), foi obtida uma média de 20,725 mm, obtendo-se o maior valor encontrado de $31,1 \mathrm{~mm}$ e o menor de $9,4 \mathrm{~mm}$.

Os resultados obtidos na medida do forame da mandíbula em relação ao côndilo, gônio, bordas anterior e posterior do lado esquerdo estão resumidos na Figura 3. Observa-se também que não houve variação significativa entre os valores de média e mediana, o que demonstra uma baixa dispersão dos resultados, com distribuição simétrica dos valores. Além disso, os valores mais elevados de desvio padrão foram encontrados nas medidas de distância entre o forame da mandíbula e o gônio esquerdo e os valores mais baixos foram aqueles obtidos nas medidas entre o forame e a borda posterior esquerda, conforme observado na Tabela 1.

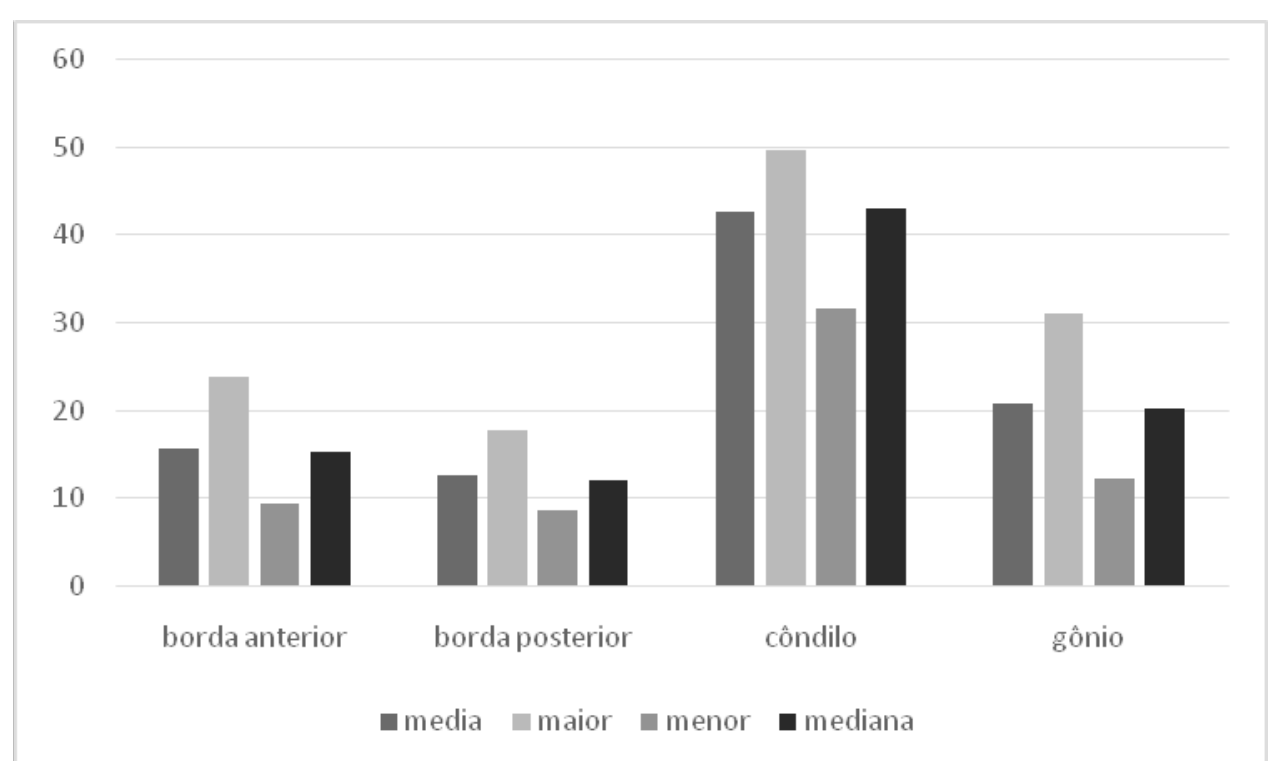

Figura 3. Resultados obtidos na medida do forame da mandíbula em relação ao côndilo, gônio, borda anterior e borda posterior do lado esquerdo.

Na Tabela 1 são agrupados os valores médios obtidos nas análises das mandíbulas, comparando-se os valores de ambos os lados. A maior variação foi observada nas medições da borda anterior, com variação de cerca de $1,2 \%$.

As demais variações foram menores que $1 \%$ e não se mostraram significativas.

$\mathrm{Na}$ análise individual dos antímeros direito e esquerdo, pode-se observar que as medidas da borda posterior direita foram as que apresentaram menor valor de desvio padrão, o que significa que os resultados estão mais próximos da média. Por outro lado, a distância ao gônio direito foi a que apresentou o maior desvio padrão, mostrando-se mais distante do valor médio obtido. Os valores mais elevados de desvio padrão foram encontrados nas medidas de distância entre o forame da mandíbula e o gônio esquerdo e os valores mais baixos foram aqueles obtidos nas medidas entre o forame e a borda posterior esquerda.

Após finalizada a mensuração de todos os pontos foi feita a análise de variância, utilizando o teste Anova, no qual comparou-se as médias dos resultados para decidir se as diferenças são reais (significativas) ou casuais (mera variabilidade). Ao final da comparação os resultados demonstraram que não houve diferenças reais. 
Tabela 1. Resultados obtidos da média (mm), desvio padrão e variação das medidas obtidas do forame da mandíbula em relação ao côndilo, gônio, borda anterior e borda posterior de ambos os lados

\begin{tabular}{lcc}
\hline & $\begin{array}{c}\text { Média (mm) e } \\
\text { desvio padrão } \\
\text { Lado Direito }\end{array}$ & $\begin{array}{c}\text { Média (mm) e } \\
\text { desvio padrão } \\
\text { Lado Esquerdo }\end{array}$ \\
\hline Borda Anterior & $15,3 \pm 2,87$ & $15,5 \pm 3,86$ \\
Borda Posterior & $12,5 \pm 2,52$ & $12,6 \pm 2,43$ \\
Côndilo & $42,8 \pm 3,95$ & $42,7 \pm 4,07$ \\
Gônio & $20,8 \pm 4,25$ & $20,7 \pm 4,62$ \\
\hline
\end{tabular}

Fonte: Dados da pesquisa.

\section{DISCUSSÃO}

Foram analisadas 48 mandíbulas humanas secas e os dados encontrados foram confrontados com outras pesquisas, as quais tinham os mesmos propósitos de encontrar as médias e variações das medidas em relação ao forame da mandíbula para saber se há variações anatômicas significantes no forame da mandíbula que justifiquem falhas nas técnicas de bloqueio anestésico do nervo alveolar inferior. Os resultados apontam que não houve variações anatômicas significativas de localização do forame da mandíbula em relação aos pontos de referência localizados no ângulo e no ramo da mandíbula, mesmo quando consideradas mandíbulas dentadas, semidentadas e adentadas.

Os dados foram testados utilizando a análise de variância pelo Anova e não foi possível detectar diferença significativa entre as amostras.

Em estudo existente no Brasil, conforme Minatel (2008), feito na Faculdade de Medicina de Jundiaí (SP), foram realizadas medidas de 177 mandíbulas de cadáveres adultos, sendo encontradas as seguintes medidas médias: ponto condílico direito $15,30 \mathrm{~mm}$ e esquerdo $19,96 \mathrm{~mm}$; ponto gônio direito $22,30 \mathrm{~mm}$ e esquerdo $22,56 \mathrm{~mm}$; borda anterior direita $17,00 \mathrm{~mm}$ e esquerda $18,06 \mathrm{~mm}$; e borda posterior direita $12,50 \mathrm{~mm}$ e esquerda $11,90 \mathrm{~mm}$

Os dados encontrados para as médias do forame da borda anterior foram de $15,537 \mathrm{~mm}$ para o lado esquerdo e 15,285 mm, não demonstrando significativas variações entre os antímeros. Apesar dos resultados mostrarem uma média menor do que aquela encontrada por Oguz e Bokir (2002) que, ao analisar 34 mandíbulas, teve como média $16,78 \mathrm{~mm}$ para a borda anterior esquerda e 16,90 mm para a direita. Já os resultados apresentados por Lima et al. (2011) mostram dados médios com valores de 19,96 mm para borda anterior esquerda e 19,48 mm para a direita. Mesmo que haja diferenças de médias encontradas entre os autores, com relação à medida da borda anterior, todos apresentam médias semelhantes entre os antímeros, estando de acordo com nossos resultados.

A distância média encontrada para a borda posterior foi de $12,593 \mathrm{~mm}$ para a borda esquerda e de $12,531 \mathrm{~mm}$ para a direita, estando de acordo com aquelas encontradas no trabalho de Minatel (2008), que obteve a média de 11,90 mm para o lado esquerdo da borda posterior e $12,50 \mathrm{~mm}$ para o lado direito, uma média significativamente menor que o do trabalho de Lima et al. (2011), onde foram encontrados $17,79 \mathrm{~mm}$ para o lado esquerdo e 17,61 mm para o direito, confirmando, assim, que a borda posterior do forame da mandíbula sempre apresenta medidas menores que a anterior, conforme demonstrado na Tabela 2 .

Segundo Strini et al. (2006), para se localizar o forame da mandíbula pode-se utilizar a língula da mandíbula como referência pois ela ocupa uma posição póstero-superior na face interna do ramo da mandíbula, estando geralmente mais próxima da borda posterior.

Para a distância entre o forame do seu ponto mais inferior até o ponto gônio, para o lado esquerdo, obtevese um valor médio de $20,725 \mathrm{~mm}$ e, para o lado direito, 20,762 mm, aproximando-se dos dados encontrados por Minatel (2008), que encontrou uma média de 22,56 mm para o lado esquerdo e $22,30 \mathrm{~mm}$ para o lado direito. Em ambos os casos, foi encontrada uma diferença significativa em comparação com o trabalho de Oguz e Bokir (2002), que encontraram valores de $29,75 \mathrm{~mm}$ para o lado esquerdo e $30,97 \mathrm{~mm}$ para o direito.

Os trabalhos dos autores Oguz e Bozkir (2002), Minatel (2008), Lima et al. (2011) mostram que foi mensurada a média entre o forame da mandíbula e a incisura da mandíbula. Neste trabalho, em especial, foi eleita a distância mais superior do forame até o ponto mais distante da cabeça do côndilo da mandíbula, tomando em conta que poderia haver alguma relação 
na variação anatômica do forame da mandíbula e algum desgaste que fosse gerado por problemas na articulação temporo-mandibular, assim como desgaste na cabeça do côndilo, encontrando uma média de 42,662 mm para o lado esquerdo e de $42,839 \mathrm{~mm}$ para o lado direito, como observado na Tabela 2.

Tabela 2. Valores médios obtidos em mm, em trabalhos com medidas do forame da mandíbula em relação ao gônio, à borda anterior e à borda posterior

\begin{tabular}{lccccccc}
\hline & $\begin{array}{c}\text { Número de } \\
\text { mandíbulas } \\
\text { analisadas }\end{array}$ & $\begin{array}{c}\text { Borda } \\
\text { anterior } \\
(\mathrm{esq}), \mathrm{mm}\end{array}$ & $\begin{array}{c}\text { Borda } \\
\text { anterior } \\
(\text { dir) }), \mathrm{mm}\end{array}$ & $\begin{array}{c}\text { Borda } \\
\text { posterior } \\
\text { (esq), mm }\end{array}$ & $\begin{array}{c}\text { Borda } \\
\text { posterior } \\
\text { (dir), mm }\end{array}$ & $\begin{array}{c}\text { Gônio } \\
\text { (esq), } \\
\text { mm }\end{array}$ & $\begin{array}{c}\text { Gônio } \\
(\text { dir), mm }\end{array}$ \\
\hline AUTOR (2015) & 48 & 15,537 & 15,285 & 12,593 & 12,531 & 20,725 & 20,762 \\
LIMA et al. (2011) & 38 & 19,96 & 19,48 & 17,79 & 17,61 & 24,97 & 24,27 \\
MINATEL (2008) & 177 & 18,06 & 17,00 & 11,90 & 12,50 & 22,56 & 22,30 \\
OGUZ e BOZKIR (2002) & 34 & 16,78 & 16,90 & 14,37 & 14,09 & 29,75 & 30,97 \\
\hline
\end{tabular}

Fonte: Dados da pesquisa.

Dados de mediana e desvio padrão apontam poucas variações em relação ao lado direito e esquerdo nas mandíbulas avaliadas, identificando pouca assimetria na localização de ambos os lados, o que sugere que existe uma maior desproporção entre os indivíduos estudados do que entre os respectivos lados. Nossos resultados vêm ao encontro à descrição de Strini et al. (2006), quando enfatizam que a maioria das falhas anestésicas do nervo mandibular inferior provem da falta do conhecimento anatômico como ponto de referência para administrar o anestésico, além de variações que podem ser encontradas como inervação acessória da dentição mandibular, dos nervos milo- hióideo, lingual, bucal.

Para o sucesso no bloqueio anestésico existem várias técnicas para realizar tal procedimento $\mathrm{e}$, em todas elas, o conhecimento anatômico é essencial para o sucesso do mesmo. Porém, muitas vezes, mesmo com o conhecimento anatômico adequado, as técnicas anestésicas parecem não surtir um bom efeito. Nesses casos se faz de extrema relevância considerar as variações anatômicas, conforme afirmam Andrade et al. (2015), que no caso específico deste trabalho faz com que o forame da mandíbula esteja deslocado mais anteriormente, mais posteriormente, mais superiormente ou mais inferiorizado. Em todos estes casos, por mais que haja difusão do sal anestésico pelos tecidos, a distância entre o forame e o local da aplicação pode dificultar a efetividade do procedimento, não causando assim um bloqueio efetivo do nervo alveolar inferior (NERY DE LIMA; CÉSPEDES, 2009).
Além das variações anatômicas é importante também considerar como fator das possíveis falhas em anestesias odontológicas a compreensão farmacológica e suas respectivas indicações. As propriedades físicoquímicas de cada anestésico local determinam a ação, potencialidade e duração da anestesia. Logo, a compreensão dos aspectos farmacológicos dos anestésicos locais é importante para a seleção da droga a ser utilizada em cirurgia (PAIVA; CAVALCANTI, 2005).

\section{CONCLUSÃO}

Não houve variações significativas nas medidas do forame da mandíbula em relação ao côndilo, gônio, borda anterior e borda posterior em ambos os lados. Em todos os casos, as variações entre os antímeros foram menores que os valores de desvio padrão encontrados, o que sugere que existe uma maior desproporção entre os indivíduos estudados do que entre os respectivos lados.

Contudo, pode-se verificar que em geral as falhas recorrentes em anestesia para bloqueio do nervo alveolar inferior dificilmente podem ser atribuídas à variação anatômica do forame da mandíbula ou pela presença de forames acessórios, sendo que o problema geralmente deve ocorrer por causas relacionadas à técnica anestésica utilizada, desconhecimento anatômico da região e ação específica dos fármacos. 


\section{AGRADECIMENTOS}

Ao laboratório de Anatomia Humana da Universidade Estadual de Maringá.

\section{REFERÊNCIAS}

AMORIM, M. M. Descrição morfológica do canal mandibular em radiografias panorâmicas. 2006. 40f. Dissertação (Mestrado em Biologia Buco-dental) Faculdade de Odontologia de Piracicaba, Universidade Estadual de Campinas, Piracicaba (SP).

ANDRADE, Y. D. N.; ARAUJO, E. B. D. J.; SOUZA, L. M. D. A.; GROPPO, F. C. Análise das variações anatômicas do canal da mandíbula encontradas em radiografias panorâmicas. Rev Odontol UNESP, v. 44, n. 1, p. 31-36, 2015.

DI DIO, L. J. A. Tratado de anatomia sistêmica aplicada. Rio de Janeiro: Atheneu, 2002. p. 120. v. 1.

FERNANDEZ, C.; READER, A.; BECK, M.; NUSSTEIN, J. A prospective, randomized, double-blind comparison of bupivacaine and lidocaine for inferior alveolar nerve blocks. J. Endod. v. 31, n. 7, p. 499-503. 2005.

GARDNER, E.; GRAY, D. J.; O'RAHILLY, R. Anatomia: estudo regional do corpo humano. $4^{\mathrm{a}}$ ed. Rio de Janeiro: Guanabara Koogan, 1988. p. 726-729.

LIMA, D. S. C.; ANDRADE, A.; FIGUERÊDO, E. A.; ROCHA, V. H.; COSTA, M. V.; CASTRO, M. P.; SILVA, R. C. P.; ARAÚJO, L. P.; MENDONÇA, V. R. R.; GRAVINA, P. R.; MENESES, J. V. L. Estudo anatômico do forame mandibular e suas relações com pontos de referência do ramo da mandíbula. Rev. Bras. Cir. Craniomaxilofac. v. 14, n. 2, p. 91-96, 2011.

MADEIRA, M. C. Anatomia da face: bases anatomos funcionais para a prática odontológica. 7. ed. São Paulo: Sarvier, 2010, 272 p.

MINATEL, E.; RANDI, B. A.; FERRAGUTI, J. M.; CALDEIRA, E. J. Análise métrica da localização do forame mandibular. Perspectivas Médicas, v. 19, n. 1, p. 20-22, 2008.
NERY DE LIMA, A.; CÉSPEDES, I. C. Fatores que levam ao sucesso da anestesia Pterigomandibular. Rev Odonto, São Bernardo do Campo, v. 17, n. 33, 2009.

OGUZ, O.; BOZKIR, M. G. Evaluation of location of mandibular and mental foramina in dry, young, adult human male, dentulous mandibles. West Indian Med J., v. 51, n. 1, p. 14-16, 2002.

PAIVA, L. C. A.; CAVALCANTI, A. L. Anestésicos locais em odontologia: Uma revisão de literatura. C. Biol. Saúde, Ponta Grossa, v. 11, n. 2, p. 35-42, jun. 2005.

QUEIROZ, S. B. F.; MAGRO FILHO, O.; LIMA, V. N.; STATKIEVICZ, C.; BONARDI, J. P.; MARTINS, M. M. Eficácia da técnica de bloqueio do nervo alveolar inferior. Arch. Health Invest., v. 4, n. 5, p. 22- 27, 2015.

STRINI, P. J. S. A.; SILVA JÚNIOR, W.; RODRIGUES, D. A.; STRINI, P. J. S. A.; GUIMARÃES, E. C.; BERNARDINO JÚNIOR, R. Avaliação topográfica do forame mandibular em peças anatômicas maceradas parcialmente dentadas e edêntulas. Rev. Fac. Odontol. Univ. Passo Fundo, v. 11, n. 2, p. 11-15, 2006.

SUAZO, I. C. G.; HERRERA, C. A. M.; LÓPEZ, M. G. C.; MATAMALA, D. A. Z. Aspectos biometricos del canal mandibular. Int. J. Morph., v. 25, p. 811-816, 2007.

VASCONCELOS, B. C. D. E.; SILVA, L. C. F. D.; SILVA FILHO, J. P. D.; MAURÍCIO, H. D. A.; VASCONCELOS, C. F. D. M. Aspirações Positivas em Anestesias por Bloqueio do Nervo Alveolar Inferior. Revista Cir. Traumatol. BucoMaxilo-Fac., v. 7, p. 19-24, 2008.

Recebido em: 29 de fevereiro de 2016 Versão final recebida em: 29 de abril de 2016 Aceito em: 03 de maio de 2016 